\title{
Astrophysical Applications of Approximate Methods for Molecular Opacities \\ - from stars to substellar brown dwarfs
}

\author{
Takashi Tsuji \\ Institute of Astronomy, The University of Tokyo, Mitaka, Tokyo, 181 Japan
}

\section{Introduction}

For studies of radiative processes in stellar environment, knowledges on opacities are essential, and molecular line opacities play a dominant role in many cases where temperatures are roughly below 4,000K. Accurate treatments of molecular line opacities require high precision line data for millions (and up to billions?) of lines. Recent progress in theoretical as well as in experimantal molecular physics made it possible to realize such an approach, and dedicated efforts are being done to developing extensive databases for polyatomic (Jørgensen in this volume) as well as for diatomic (Kurucz in this volume) molecules. These accurate databases should be of fundamental importance in our understanding of astronomical observations that are progressing rapidly both in scope and in accuracy.

Once the database includes so many lines, it can be regarded as a statistical sample, and actual use of such a database generally relies on statistical treatments such as the opacity distribution function (ODF) method or the opacity sampling (OS) method. Although these treatments are more or less approximate in nature, they are regarded to be reasonably accurate and used as the standard methods for line opacities. Then, by approximate methods for molecular opacities in this review, we restrict ourselves to such treatments as the band model methods in which detailed line-by-line databases are not referred to at all. In fact, it is known that simple analytical expressions for approximate molecular line opacities can be formulated with only a few spectroscopic constants including the band $f$-values (Sect.2).

One major role of approximate opacities has been to explore new problems in which molecular opacities play a major role, yet accurate opacity data are not yet available. For example, when infrared observations in the 1960's revealed a wealth of molecular bands such as of $\mathrm{H}_{2} \mathrm{O}$ and $\mathrm{CO}$ in cool stars for the first time, it was immediately recognized that the molecular opacities play a dominant role in determining the atmospheric structures of cool stars, by the use of simple straight mean (Tsuji 1967) or harmonic mean (Auman 1967a) opacities. Then, somewhat improved methods such as the regular band model (Tsuji 1969) or more sophisticated random band model (Alexander et al. 1989) have been intro- 
duced. Apart from such exploratory purposes, approximate opacities are quite useful in practical applications so far as they are used with their limitations in mind. For example, the band model opacities may suffice for determining the thermal structure of stellar atmospheres for many practical purposes, while they may be not accurate enough for analyzing the stellar emergent spectra in general (Sect.3).

Although accurate molecular opacities are being developed for some molecules of astrophysical interests, explorations of model atmospheres in which molecular opacities play a dominant role may still be pursued. One good example of such a new exploration may be the case of brown dwarfs. In fact, at the very high density and low temperature environment to be expected in the atmospheres of brown dwarfs, some new opacity problems will appear. For example, methane $\left(\mathrm{CH}_{4}\right)$ opacity will play an important role but, as accurate opacity for $\mathrm{CH}_{4}$ is still not available, approximate opacity will play a major role in exploring brown dwarf atmospheres (Sect.4).

Now, even if exploration of model atmospheres in the classical sense may come near the end by the brown dwarf atmospheres, this fact does not imply that exploration of stellar atmospheres themselves have also finished. It is true that the classical model atmospheres may now come to near the completion by the use of accurate opacities, and it is no doubt that this is an important achievement by itself. At the same time, such accurate classical models revealed the limitation of the classical atmospheres themselves. Especially, recent observations revealed various dynamical and non-thermal phenomena in the atmospheres of cool stars. As the classical model atmosphere is essentially a static equilibrium model, it is beyond the scope of the classical models to handle these phenomena. Certainly, for better understanding of stellar atmospheres, it is needed to understand the dynamical structure through the sub-photospheric convective zone to the outer atmosphere in details. For analyses of radiative processes together with hydrodynamics in the atmospheres of cool stars, molecular opacities will play an imp ortant role. Here again, the simplicity of approximate molecular opacities should be very attractive, since it will give more room for other thinking in an exploratory stage (Sect.5).

By the reasons outlined above, there may be sufficient reasons why we pay some attention to approximate methods for molecular opacities, even in the welcome circumstances at present when accurate molecular opacities are being developed.

\section{Basic Formulation of Band Model Methods}

\subsection{Regular and Random Band Models}

The band model method for molecular opacities was originally introduced for treating atmospheric transmission in meteorology (e.g. Goody 1964). The purpose was to provide a relatively simple representation of the mean value of the spectral transmission for a spectral mesh including many spectral lines. Also, 
the band model method has been applied for other purposes such as analyses of laboratory gas emissivities. Probably, the band model method is somewhat analogous to the classical theory of curve-of-growth familiar to astronomers: the band model method is to evaluate a growth of transmission (or emissivity) under the presence of multiple lines, while the curve-of-growth method is to analyze a growth of absorption for a single line. Since the radiative transfer problems in stellar atmospheres include much more complicated cases than just to obtain transmissions, the major techniques of the band model method cannot directly be applicable to astrophysical problems, just as the classical theory of curve-ofgrowth cannot to modern problems of spectral line formations.

For astrophysical purpose, what is useful may be the basic idea of how to model the spectral absorption coefficients for a large number of lines. For this purpose, we consider two models: one is the regular model and the other is the random model. The regular band model consists of an array of lines of equal intensity spaced at equal intervals, and such a model is known as the Elsasser model. This model can be characterized by two parameters - the mean absorption coefficient $(\bar{k})$ and the mean line separation $(\bar{d})$ - for each spectral mesh of appropriate interval, once the spectral line shape is defined. The case of the Voigt profile has been formulated by Golden (1969) and the result is known as the Voigt-Analogue Elsasser Band Model (VAEBM). It is straight forward to obtain the ODF for this model, since the same spectral shape of the same intensity repeats at the equal intervals. Roughly speaking, the ODF for this model is a Voigt profile itself truncated at the mid-point between the two adjacent lines, plus the contributions of line wings of nearby lines in the array.

On the other hand, the random band model consists of randomly spaced lines of different intensities. For example, the probability $P(S) d S$ that a line has an intensity between $S$ and $S+d S$ can be assumed as

$$
P(S)=\exp \left(-S / S_{o}\right) / S_{0},
$$

where $S_{0}$ is the mean line intensity. Also, if a sufficiently large number of lines are randomly placed in a spectral mesh, the probability that there is a gap of $x$ is given by

$$
p(x)=\exp (-x / \bar{d}),
$$

where $\bar{d}$ is the mean line separation (Goody 1964). Recently, such a model has been applied to generate the ODF for $\mathrm{H}_{2} \mathrm{O}$ by Alexander et al. (1989). For this purpose, synthetic spectrum is generated, in which the line position of the next line can be determined by Eq.(2) using a random number generator and the strength of this line by Eq.(1) again using a random number generator. Then, the OS or the ODF method can be applied to this somewhat fictitious spectrum. This is an interesting attempt, but requires as much effort as to generate a more realistic spectrum based on a line-by-line database. It is to be noted, however, that this model is also characterized by two parameters - the mean absorption coefficient $\bar{k}$ and the mean line separation $\bar{d}$ (note that $S_{0}=\bar{k} \bar{d}$ ). 


\subsection{Band Model Parameters}

Rotation-Vibration Bands of Simple Molecules For linear (and spherical top) molecules, the rotational term value is given by

$$
F_{v}(J)=B_{v} J(J+1),
$$

with the rotation constant $B_{v}$. Then, line positions for the $P$ - and $R$-branches are given by a single formula as

$$
\omega=\omega_{v^{\prime} v^{\prime \prime}}+\left(B_{v}^{\prime}+B_{v}^{\prime \prime}\right) m+\left(B_{v}^{\prime}-B_{v}^{\prime \prime}\right) m^{2},
$$

where $\omega_{v^{\prime}} v^{\prime \prime}$ is the wavenumber of the band origin, $m=-J$ for the $P$-branch and $m=J+1$ for the $R$-branch. This equation can be solved for $m$ as

$$
m=\left[-\left(B_{v}^{\prime}+B_{v}^{\prime \prime}\right) \pm \sqrt{\left(B_{v}^{\prime}+B_{v}^{\prime \prime}\right)^{2}+4\left(B_{v}^{\prime}-B_{v}^{\prime \prime}\right)\left(\omega-\omega_{v^{\prime} v^{\prime \prime}}\right)}\right] / 2\left(B_{v}^{\prime}-B_{v}^{\prime \prime}\right)
$$

The integrated line intensity for a single rotational line can be given by

$$
\begin{aligned}
S_{v^{\prime} v^{\prime \prime}}(m)= & \frac{\pi e^{2}}{m_{e} c^{2}} f_{v^{\prime} v^{\prime \prime}} \frac{\omega}{\omega_{v^{\prime} v^{\prime \prime}}}|m| F(m) \exp \left[-\frac{h c}{k T}\left\{G_{v}^{\prime \prime}+B_{v}^{\prime \prime}|m(m-1)|\right\}\right] \\
& \left(1-e^{-h c \omega / k T}\right) / Q_{r}(T) Q_{v}(T),
\end{aligned}
$$

where $f_{v^{\prime} v^{\prime \prime}}$ is the vibrational $f$-value, $F(m)$ is the Herman-Wallis factor, $G_{v}^{\prime \prime}$ is the term value of the lower vibrational level, $Q_{r}(T)$ and $Q_{v}(T)$ are the rotational and vibrational partition functions, respectively, and other notations have their usual meanings. $S_{v^{\prime} v^{\prime \prime}}(m)$ can also be understood as $S_{v^{\prime} v^{\prime \prime}}(\omega)$ by means of Eq.(5).

The line separation in the $\left(v^{\prime}, v^{\prime \prime}\right)$ band is given by

$$
d_{v^{\prime} v^{\prime \prime}}(m)=\frac{d \omega}{d m}=\left(B_{v}^{\prime}+B_{v}^{\prime \prime}\right)+2\left(B_{v}^{\prime}-B_{v}^{\prime \prime}\right) m .
$$

By applying Eq.(5), this can also be written (for each of the $P$ - and $R$-branches) as

$$
d_{v^{\prime} v^{\prime \prime}}(\omega)=\sqrt{4\left(B_{v}^{\prime}-B_{v}^{\prime \prime}\right)\left(\omega-\Omega_{v^{\prime} v^{\prime \prime}}\right)},
$$

where $\Omega_{v^{\prime}} v^{\prime \prime}$ is the wavenumber of the $\left(v^{\prime}, v^{\prime \prime}\right)$ band head and is given by

$$
\Omega_{v^{\prime} v^{\prime \prime}}=\omega_{v^{\prime} v^{\prime \prime}}-\left(B_{v}^{\prime}+B_{v}^{\prime \prime}\right)^{2} / 4\left(B_{v}^{\prime}-B_{v}^{\prime \prime}\right) .
$$

For the $Q$-branch (perpendicular band), the line position is given by

$$
\omega=\omega_{v^{\prime} v^{\prime \prime}}+\left(B_{v}^{\prime}-B_{v}^{\prime \prime}\right) m(m+1),
$$

where $m=J$. As for the $P$ - and $R$ - branches, the line separation is given by

$$
d_{v^{\prime} v^{\prime \prime}}(\omega)=\sqrt{4\left(B_{v}^{\prime}-B_{v}^{\prime \prime}\right)\left[\omega-\omega_{v^{\prime} v^{\prime \prime}}+\left(B_{v}^{\prime}-B_{v}^{\prime \prime}\right) / 4\right]} .
$$



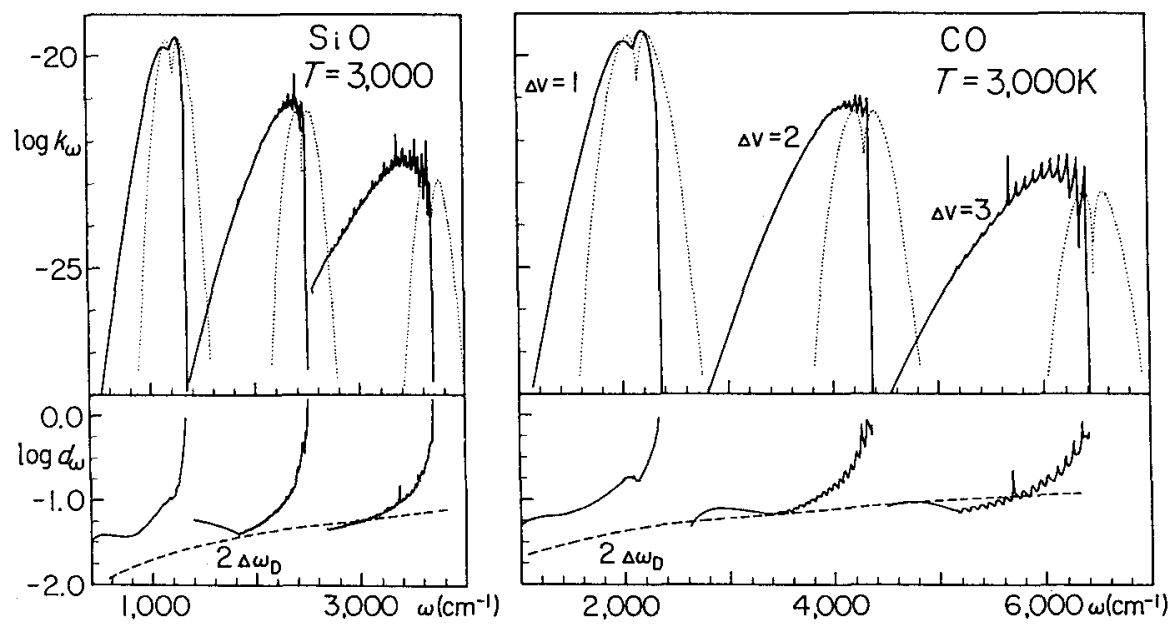

Fig. 1. The absorption cross-sections $\bar{k}\left(\mathrm{~cm}^{2} /\right.$ molecule) and the mean line separations $\bar{d}\left(\mathrm{~cm}^{-1}\right)$, averaged over $10 \mathrm{~cm}^{-1}$, for $\mathrm{SiO}$ and $\mathrm{CO}$ at $T=3,000 \mathrm{~K}$. The results of the rigid rotator harmonic oscillator (RRHO) model are shown by the dotted lines. The full Doppler widths corresponding to $v_{\text {mic }}=3 \mathrm{~km} \mathrm{~s}^{-1}$ are indicated by the dashed lines.

Note that $\Omega_{v^{\prime} v^{\prime \prime}}$ in Eq.(8) is replaced by $\omega_{v^{\prime} v^{\prime \prime}}$ in Eq.(11), if we neglect a small term in Eq.(11) by utilizing the fact that $\left(\omega-\omega_{v^{\prime} v^{\prime \prime}}\right) \gg\left(B_{v}^{\prime}-B_{v}^{\prime \prime}\right) / 4$.

Now, we follow the standard definitions of the band model parameters. First, the weak line parameter is defined by

$$
\left(\frac{S}{d}\right)=\sum_{v^{\prime} v^{\prime \prime}}\left[\frac{S_{v^{\prime} v^{\prime \prime}}(\omega)}{d_{v^{\prime} v^{\prime \prime}}(\omega)}\right]
$$

where the summation should be extended to all the bands that are contributing at $\omega$. The resulting $(S / d)$ is nothing but the straight mean absorption coefficient and can be denoted as $\bar{k}(\omega)$. Second, the strong line parameter is defined by

$$
\left(\frac{S^{1 / 2}}{d}\right)=\sum_{v^{\prime} v^{\prime \prime}}\left[\frac{S_{v^{\prime} v^{\prime \prime}}(\omega)^{1 / 2}}{d_{v^{\prime} v^{\prime \prime}}(\omega)}\right]
$$

Then, we defined

$$
\left(\frac{1}{d}\right)=\left(\frac{S^{1 / 2}}{d}\right)^{2} /\left(\frac{S}{d}\right),
$$

which provides the effective mean line separation $\bar{d}(\omega)$. As $1 / d_{v^{\prime}} v^{\prime \prime}$ is the number of lines of the $\left(v^{\prime}, v^{\prime \prime}\right)$ band in $1 \mathrm{~cm}^{-1}$, a more naive definition of $\bar{d}$ may be

$$
\left(\frac{1}{\bar{d}}\right)=\sum_{v^{\prime} v^{\prime \prime}} \frac{1}{d_{v^{\prime} v^{\prime \prime}}(\omega)} .
$$




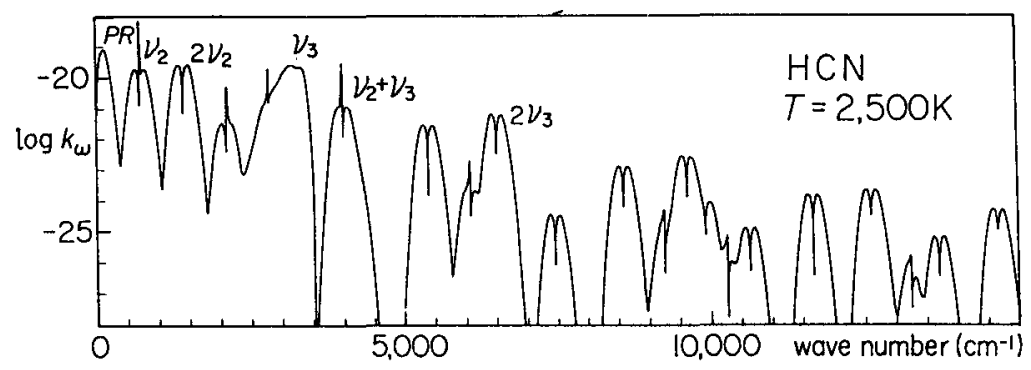

Fig. 2. The absorption cross-sections $\bar{k}\left(\mathrm{~cm}^{2} /\right.$ molecule) of $\mathrm{HCN}$ for 28 band systems including the pure rotation band, at $T=2,500 \mathrm{~K}$. The RRHO model is applied, except for the $\nu_{3}$-band system for which about 1,000 bands are considered band-by-band.

In this case, however, we must introduce a cutting criterion, since $\bar{d}$ may be unrealistically small if we consider very weak bands in the summation.

If we introduce the rigid-rotator-harmonic-oscillator (RRHO) model, the straight mean absorption coefficient defined by Eq.(12) can be reduced to a simple analytical formula and it is no longer necessary to do summations bandby-band (e.g. Tsuji 1984). This model provides a large simplification especially in the case of polyatomic molecules, but the shape of the absorption coefficient as a function of wavenumber cannot be represented very well if the effects of non-rigidity and anharmonicity are important (see Fig.1).

As examples of diatomic molecules, the band model parameters are shown in Fig. 1 for $\mathrm{CO}$ and $\mathrm{SiO}$. Also shown are the cases of the RRHO model, which appear to be a poor approximation to the detailed band-by-band approach especially for overtone bands. Also, it is shown that the mean line separations are generally larger than the full Doppler widths for turbulent velocity of $3 \mathrm{~km}$ $\mathrm{s}^{-1}$. As an example of polyatomic linear molecules, the straight mean absorption cross-sections for HCN are shown in Fig.2. The mean line separations are generally smaller than the Doppler widths for appropriate turbulent velocity as shown in our previous analysis (Tsuji 1984), where only a limited number of band systems were included. Here, we also included the pure rotation transitions, which are already important in the mid-infrared at high temperatures.

Another molecule of potential importance at high density and low temperature environment is $\mathrm{CH}_{4}$, since $\mathrm{CO}$ is no longer the most stable molecule, and most carbon atoms will be in $\mathrm{CH}_{4}$ at temperatures below $1,000 \mathrm{~K}$ and at high densities, even in the oxygen-rich environment. $\mathrm{As}^{\mathrm{CH}_{4}}$ is a spherical top molecule, its line positions can be given by Eq.(4) for rotation-vibration transitions, and the formulation of the band model outlined above can also be applied to $\mathrm{CH}_{4}$. Only slight modification is necessary to accomodate the fact that the statistical weight of a rotational level with the quantum number $J$ is $(2 J+1)^{2}$ rather than $2 J+1$ (Herzberg 1945). The resulting straight mean absorption cross-sections of $\mathrm{CH}_{4}$ are shown in Fig.3 together with those of $\mathrm{H}_{2} \mathrm{O}$ based on the experimental data by Ludwig (1967). It is interesting to notice that $\mathrm{CH}_{4}$ bands nicely cover 


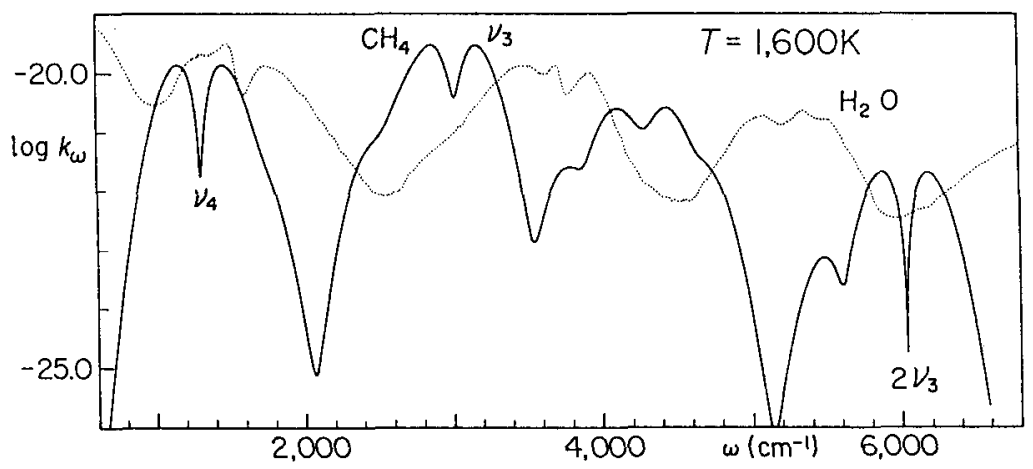

Fig. 3. The absorption cross-sections $\bar{k}\left(\mathrm{~cm}^{2} /\right.$ molecule $)$ of $\mathrm{CH}_{4}$ at $T=1,600 \mathrm{~K}$, including 14 band systems by the RRHO model, and those of $\mathrm{H}_{2} \mathrm{O}$ (Ludwig 1967) for comparison.

the regions that are least absorbed by $\mathrm{H}_{2} \mathrm{O}$. Thus, the infrared spectral region is completely opaque if these two species exist together and such a situation will actually be realized in the atmospheres of brown dwarfs (Sect.4).

Electronic Bands of Diatomic Molecules More or less similar consideration can be applied to electronic bands of diatomic molecules. With an approximation that no distinction is made between the $P_{-}, Q_{-}$, and $R$-branches, the straight mean absorption cross-section has been given by Golden (1967) as

$$
\begin{aligned}
k_{v^{\prime} v^{\prime \prime}}(\omega)= & \frac{\pi e^{2}}{m_{e} c^{2}} f_{v^{\prime} v^{\prime \prime}} \frac{1}{\left|B_{v}^{\prime}-B_{v}^{\prime \prime}\right|} \exp \left[-\frac{h c}{k T}\left\{G_{v}^{\prime \prime}+\frac{B_{v}^{\prime \prime}}{B_{v}^{\prime}-B_{v}^{\prime \prime}}\left(\omega-\Omega_{v^{\prime} v^{\prime \prime}}\right)\right\}\right] \\
& g_{e}^{\prime \prime} \exp \left(-\frac{h c}{k T} T_{e}^{\prime \prime}\right)\left(1-e^{-h c \omega / k T}\right) / Q_{r}(T) Q_{v}(T) Q_{e}(T),
\end{aligned}
$$

where $Q_{e}(T)$ is the electronic partition function, $g_{e}^{\prime \prime}$ and $T_{\varepsilon}^{\prime \prime}$ are the statistical weight and the term value of the lower electronic state, respectively. If the electronic dipole moment is independent of the internuclear distance, the band oscillator strength $f_{v^{\prime} v^{\prime \prime}}$ can be given by

$$
f_{v^{\prime} v^{\prime \prime}}=f_{e} \frac{\omega_{v^{\prime} v^{\prime \prime}}}{\omega_{00}} q_{v^{\prime} v^{\prime \prime}}
$$

where $\omega_{00}$ is the wavenumber of the band origin of the $(0,0)$ band, $f_{e}$ is the electronic oscillator strength defined at $\omega_{00}$, and $q_{v^{\prime} v^{\prime \prime}}$ is the Franck-Condon factor.

In consistency with Golden's formulation, we use Eq.(8) for the line separation throughout but, if the $P_{-}, Q-$, and $R$-branches are present, actual line separation must be 3 times smaller than that given by Eq.(8). Further, the line separation may still be smaller due to the $\Lambda$-type doubling and due to the splitting by the spin-orbital interaction. Thus, we finally have

$$
d_{v^{\prime} v^{\prime \prime}}(\omega)=\sqrt{4\left(B_{v}^{\prime}-B_{v}^{\prime \prime}\right)\left(\omega-\Omega_{v^{\prime} v^{\prime \prime}}\right)} / 3(2 S+1)\left(2-\delta_{\Lambda, 0}\right),
$$




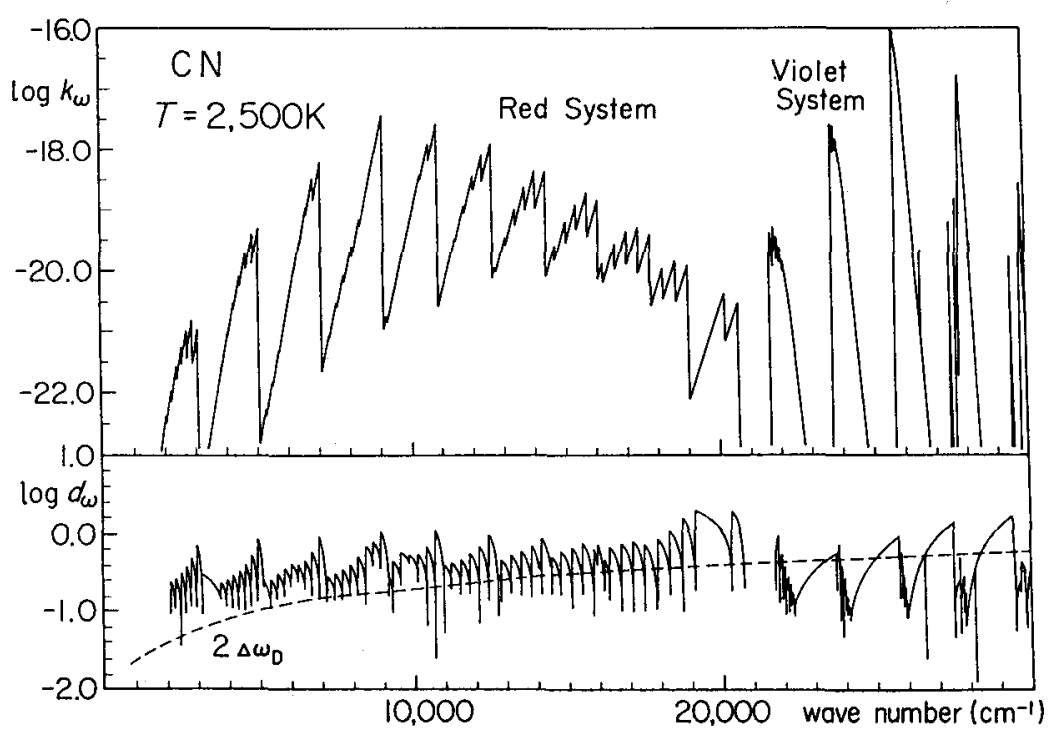

Fig. 4. The absorption cross-sections $\bar{k}\left(\mathrm{~cm}^{2} /\right.$ molecule $)$ and the mean line separations $\bar{d}\left(\mathrm{~cm}^{-1}\right)$, averaged over $10 \mathrm{~cm}^{-1}$, for the $\mathrm{CN}$ Red and Violet systems at $T=2,500 \mathrm{~K}$. The full Doppler widths corresponding to $v_{\text {mic }}=3 \mathrm{~km} \mathrm{~s}^{-1}$ are indicated by the dashed lines.

where $2 S+1$ is the spin multiplicity, $A$ is the component of the electronic angular momentum along the internuclear axis, $\delta$ is the Kronecker's delta function. The band model paremeters based on this model are shown in Fig.4 for the CN Red and Violet systems.

A formulation free from some of the simplifications used in Eq.(16) is given by Zeidler-K.T. \& Koester (1982), who treated the $P_{-}, Q-$, and $R$ - branches separately.

\subsection{Band Model Opacities for Astrophysical Applications}

Now, we have the two parameters - the straight mean absorption cross-section $\bar{k}_{i}(\omega)$ and the mean line separation $\bar{d}_{i}(\omega)$ - for each molecule that is important as an opacity source. Then, we evaluate the absorption ceofficient per gram of stellar material by solving the chemical equilibrium for given temperature and gas pressure. We now apply Eqs.(12)-(14), which were applied to different bands of the same species in Sect.2.2, to different species that give non-negligible contribution at a given spectral mesh [ note that $S_{0 i}(\omega)=\bar{k}_{i}(\omega) \bar{d}_{i}(\omega)$ for $i$ th species]. The results are the integrated mass absorption coefficient and the average mean line separation for the given spectral mesh, chemical composition, temperature, and gas pressure. Then, the ODF can easily be estimated within the framework of the VAEBM, by which line broadenings due to turbulence and collision are considered at each step of integration of the model atmosphere. 
In this way, once the two parameters $-\bar{k}_{i}(\omega)$ and $\bar{d}_{i}(\omega)$ - for each molecule are prepared, an approximate ODF can easily be estimated for any chemical composition, temperature, gas pressure, and turbulent velocity during the construction of a model atmosphere, and it is not necessary to prepare large tables of opacities covering of all the parameter space. Such simplicity and flexibility are the most attractive features of the VAEBM even at the loss of accuracy as compared with the ODF based on a detailed line-by-line database. By the way, the ODF based on a line-by-line database is not necessarily very accurate when several different species are contributing in the same spectral mesh, since the line that gives the maximum absorption is not necessarily due to the same species at different depths in stellar atmosphere. As is well known, the ODF method cannot consider such an effect, and the OS method is more consistent in this regard. On the other hand, an efficient method for calculating composite ODF's from the ODF for each individual opacity source has been developed by Saxner \& Gustafsson (1984), and it is possible to change the chemical composition without recalculation of ODF's in the construction of model atmospheres. Such a flexibility is certainly an advantage of the ODF method over the OS method.

\section{Applicability and Limitation of Approximate Opacities}

The band model opacities can further be simplified if the mean line separation is comparable to or smaller than the line width. In this case, the line absorption coefficient can be treated as a quasi-continuous absorption and is given by the straight mean $\bar{k}(\omega)$ itself. Line opacity based on this assumption is referrred to as the Just Overlapping Line Approximation (JOLA). It is in this most simple form that the molecular line opacities such as of $\mathrm{H}_{2} \mathrm{O}, \mathrm{CO}$, and $\mathrm{OH}$ have first been introduced in stellar opacities (Tsuji 1966), although it has been recognized that such an approximation may not necessarily be valid for stellar environment in general. Neverthless, such a simple opacity played an important role in the exploratory stage of constructing model atmospheres of cool stars (Sect.1). Also, harmonic mean was considered for $\mathrm{H}_{2} \mathrm{O}$ (Auman 1967b), but the reason for using the harmonic mean for atmospheric problems was not very clear.

Further, the JOLA found some useful applications. For example, the cooling effect of $\mathrm{CO}$ in the atmospheres of cool stars has been examined in detail by this simple technique by Johnson (1973). A case where the JOLA may actually be valid can be found in the atmospheres of cool white dwarfs, since molecular lines are completely smeared out by the large pressure broadening at the high densities of white dwarf atmospheres. Thus, successful applications of the JOLA have been done to model the atmospheres of $\mathrm{C}_{2}$ white dwarfs (Grenfell 1974; Wehrse 1975). Also, it is by the JOLA that the collision-induced absorption (CIA) of $\mathrm{H}_{2}$ has first been introduced in the actual computation of stellar model atmospheres (Tsuji 1969). In fact, the JOLA is well justified in this case, since individual lines are highly broadened because of the short time of the intermolecular interaction inducing the dipole moment and hence completely smeared out.

The next simple approximation - the regular band model - was introduced 


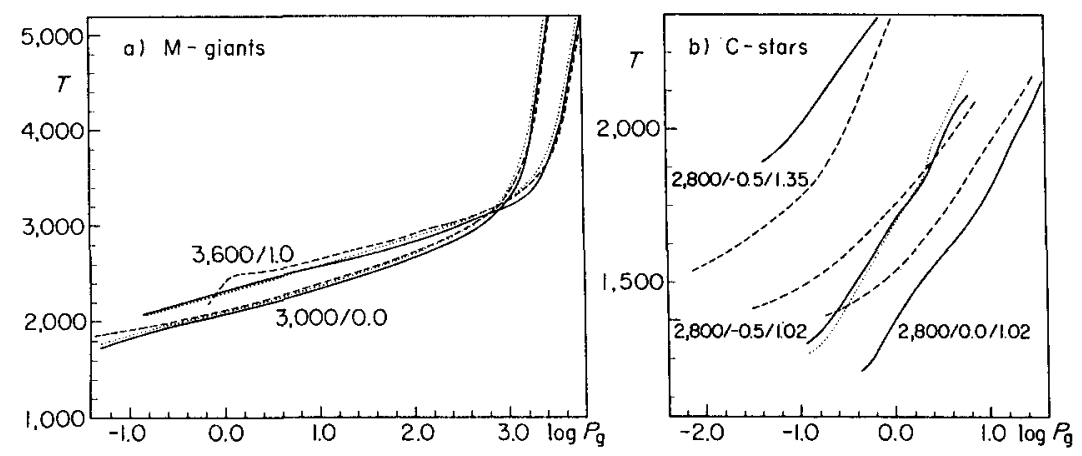

Fig. 5. a) Model atmospheres of oxygen-rich giant stars based on the VAEBM (solid lines) and on the JOLA (dotted lines) are compared with those based on the OS method (Brown et al. 1989; dashed lines). $T_{\text {eff }} / \log g$ are indicated for each model, and other input parameters are $v_{\text {mic }}=1 \mathrm{~km} \mathrm{~s}^{-1}$ and the solar composition. b) Model atmospheres of carbon stars based on the VAEBM (solid lines) and on the JOLA (dotted lines) are compared with those base on the OS method (Jørgensen et al. 1992; dashed lines). $T_{\mathrm{eff}} / \log g /(\mathrm{C} / \mathrm{O})$-ratio are indicated for each model and $v_{\text {mic }}=2 \mathrm{~km} \mathrm{~s}^{-1}$ throughout.

first for modelling the atmospheres of $\mathrm{M}$ dwarfs (Tsuji 1969). Then, the VAEBM was applied to estimating the Rosseland mean opacities of the oxygen-rich mixture (Tsuji 1971), and to constructing model atmospheres of red giant and supergiant stars (Tsuji 1976; 1978). In this VAEBM approach, the band model parameters $\left(\bar{k}_{i}\right.$ and $\left.\bar{d}_{i}\right)$ of 9 molecules including $\mathrm{H}_{2} \mathrm{O}, \mathrm{CO}, \mathrm{OH}, \mathrm{CN}, \mathrm{CH}, \mathrm{TiO}$, $\mathrm{MgH}, \mathrm{CaH}, \mathrm{SiH}$ (and $\mathrm{H}_{2} \mathrm{CIA}$ as a quasi-continuum) were given for 72 spectral meshes between 0.3 and $30 \mu \mathrm{m}$ (hereafter be referred to as VAEBM72). Here, the band model parameters for $\mathrm{H}_{2} \mathrm{O}$ were based on the experimental data by Ludwig (1967). At the same time, more elaborate treatments such as the ODF method (e.g. Querci et al. 1974; Gustafsson et al. 1975) or the OS method (e.g. Johnson et al. 1980) have been preferred in general, and the band model opacities have not necessarily been used extensively. Recently, however, an interesting attempt to apply the random band model to $\mathrm{H}_{2} \mathrm{O}$ opacity sampling has been given by Alexander et al.(1989).

Generally, the degree of success of the approximate opacities as applied to model atmospheres can be tested by comparing with the results based on more elaborate opacities (also see Carbon 1979; Jørgensen 1993). Such a test, however, is by no means easy, since it is difficult to find model atmospheres with the same input physics and data except for the treatments of opacities. As an example, we compare in Fig.5a the models of red giant atmospheres by our VAEBM72 with those by the OS method (Brown et al. 1989) based on the $\mathrm{H}_{2} \mathrm{O}$ opacity by Alexander et al.(1989). Inspection of the results reveals that the VAEBM provides model atmospheres that are very similar to those based on the OS method. Even the thermal structures of the model atmospheres based on the JOLA are not much different from those based on the VAEBM as shown in Fig.5a 
or from those based on the OS opacities as shown by Alexander et al.(1989). As another example, we compare in Fig. $5 \mathrm{~b}$ the model atmospheres of carbon stars based on the OS method (Jørgensen et al. 1992) with those based on our new VAEBM opacities in which band model parameters of $\mathrm{CO}, \mathrm{CN}, \mathrm{C}_{2}, \mathrm{HCN}$, and $\mathrm{C}_{2} \mathrm{H}_{2}$ are prepared for 194 spectral meshes between 0.2 and $45 \mu \mathrm{m}$ (hereafter be referred to as VAEBM194). Here, the agreement in temperatures is generally within $100 \mathrm{~K}$ and differential effects due to the gravity and the $\mathrm{C} / \mathrm{O}$ ratio show reasonable agreement, between the two models based on the different opacities.

A rationale for the result that the temperature structure can be relatively well determined by the approximate opacities can be found in the fact that the thermal structure of stellar atmosphere is decided by the constraint of radiative equilibrium - namely,

$$
\int_{0}^{\infty} \kappa_{\nu} B_{\nu} d \nu=\int_{0}^{\infty} \kappa_{\nu} J_{\nu} d \nu
$$

where $\kappa_{\nu}$ is the absorption coefficient including line absorption, $B_{\nu}$ is the Planck function, and $J_{\nu}$ is the mean intensity. That is, the radiative energy absorbed by a volume element (right hand side) must balance with the energy emitted (left hand side), at radiative equilibrium. Now, as

$$
\int_{0}^{\infty} \kappa_{\nu} B_{\nu} d \nu \approx \sum_{i=1}^{n} B_{\nu_{i}} \int_{\Delta \nu_{i}} \kappa_{\nu} d \nu
$$

if the frequency interval $\Delta \nu_{i}$ is small enough so that the Planck function is nearly constant, the volume emissivity does not depend on whether $\kappa_{\nu}$ is given by the straight mean, by the band models, by the ODF, or by the detailed OS, so far as the integrated absorption coefficient is the same in each frequency interval. Although $J_{\nu}$ depends on the details of $\kappa_{\nu}$, this effect is also largely tempered in the product $\kappa_{\nu} J_{\nu}$ in the integrand of the right hand side of Eq.(19). Thus, the temperature that is determined by Eq.(19) may depend only weakly on the details of $\kappa_{\nu}$. In deeper photospheric layers, backwarming effect may be overestimated by the JOLA which over-estimates the blocking of radiation by lines, but this is largely relaxed by the band model approach such as the VAEBM.

Next problem is how well the approximate opacities can predict such quantities as emergent fluxes. It is interesting to know that even the JOLA predicts the emergent fluxes blanketed by $\mathrm{H}_{2} \mathrm{O}$ within $20 \%$ of those based on the OS method for cool giant stars (Alexander et al. 1989), and this may be because the line density of polyatomic molecules such as $\mathrm{H}_{2} \mathrm{O}$ is so large that the lines are actually overlapping. Such a favourable condition is generally not met for diatomic molecules (see Figs.1 \& 4), and we examine the case of CO in the region of the fundamental VR bands. First, for a model atmosphere of an $\mathrm{M}$ dwarf star $\left(T_{\text {eff }}=2,400 \mathrm{~K}, \log g=5.0\right.$, and $\left.v_{\text {mic }}=1.0 \mathrm{~km} \mathrm{~s}^{-1}\right)$, emergent fluxes based on our VAEBM72, on the JOLA, and on the detailed OS method are shown by the solid, dotted, and dashed lines, respectively in Fig.6a, together with the continuum flux by the thin solid line. The OS flux is evaluated with the sampling interval of $0.2 \mathrm{~cm}^{-1}$ based on our ${ }^{12} \mathrm{C}^{16} \mathrm{O}$ line database $(J \leq 150$ and $v \leq 15)$, 

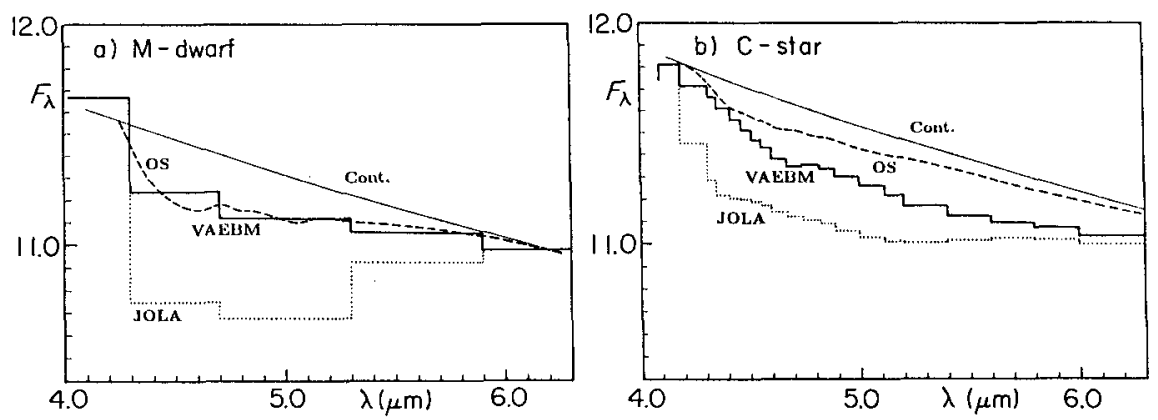

Fig. 6. Predicted emergent fluxes based on the VAEBM (solid lines) and on the JOLA (dotted lines) are compared with those base on the detailed line-by-line OS method (dashed lines), in the region blanketed by the CO fundamental. Predicted continuous flux is also shown by the thin solid line. a) For a model atmosphere of $M$ dwarf star with $\left(T_{\text {eff }} / \log g / v_{\text {mic }}\right)=\left(2,400 \mathrm{~K} / 5.0 / 1.0 \mathrm{~km} \mathrm{~s}^{-1}\right)$. b) For a model atmosphere of carbon star with $\left(T_{\text {eff }} / \log g / v_{\text {mic }}\right)=\left(3,000 \mathrm{~K} / 0.0 / 3.0 \mathrm{~km} \mathrm{~s}^{-1}\right)$.

and then averaged over interval of $30 \mathrm{~cm}^{-1}$ each. It is evident that the emergent flux based on our VAEBM72 agrees well with the accurate OS flux while that based on the JOLA shows unacceptably large deviation from the true flux. Second test is for a carbon star $\left(T_{\text {eff }}=3,000 \mathrm{~K}, \log g=0.0\right.$, and $\left.v_{\text {mic }}=3.0 \mathrm{~km} \mathrm{~s}^{-1}\right)$ with our VAEBM194, and the results are shown in Fig.6b. Here, the emergent flux based on the VAEBM194 does not agree so well with the accurate OS flux, although it provides a large improvement over that based on the JOLA. The discrepancy between the flux based on the VAEBM194 and the OS flux is larger at the longer wavelength side of the CO band where $\bar{d}$ based on Eq.(14) is also smaller (see Fig.1). This may indicate that the estimation of $\vec{d}$ based on Eq.(14) is not necessarily correct. On the other hand, $\bar{d}$ of the VAEBM72 was based on Eq.(15) with an appropriate cutting criterion (Tsuji 1976) and the result appears to be fine. Thus, how to estimate the mean line separation is crucial in the band model approach and this problem should be examined further.

Finally, it is to be remembered that the JOLA has played some roles in exploring effects of sphericity in the model atmospheres of oxygen-rich (super)giants (e.g. Watanabe \& Kodaira 1978; Schmid-Burgk et al. 1981) as well as of cool carbon stars (Scholz \& Tsuji 1984). The major physical effects have mostly been confirmed by the recent works based on the OS method both for the oxygen-rich (Pletz et al. 1992) and carbon-rich (Jørgensen et al. 1992) cases, although the sphericity effects appear to be larger in the models by the JOLA. It is true that the JOLA tends to over-estimate the effect of molecular opacities, while completeness of the line list is crucial in the OS method. This is clearly shown for the case of $\mathrm{H}_{2} \mathrm{O}$, for which such a completeness has finally been achieved (Jørgensen in this volume). The completeness can easily be realized in the JOLA only if the integrated intensities are correctly given, but this makes sense only if the JOLA is valid. This must anyhow be confirmed by a complete line database. 


\section{An Exploratory Model Atmosphere of Brown Dwarfs}

The primary role of approximate opacities is to explore new problems, as it has been in the past. One current problem to be explored is the model atmosphere of brown dwarf, which is a substellar object whose existence has been predicted by several arguments but not confirmed yet by any direct observation, in spite of extensive searches (Liebert in this volume). For further searches to be successful, it should be indispensable to understand the physical properties of the atmospheres of brown dwarfs and to predict the observable properties such as infrared spectra more carefully. As compared with the atmosphere of $\mathbf{M}$ dwarfs (Scholz \& Wehrse in this volume), a major difference is the predominance of $\mathrm{CH}_{4}$ in the atmosphere of brown dwarfs as noted in Sect.2.2. Thus, we examine the effect of methane opacity on the atmospheric structure and infrared spectra of brown dwarfs by the use of the approximate $\mathrm{CH}_{4}$ opacity discussed in Sect.2.2.

For this purpose, we extended our model atmosphere code of cool dwarf stars to be used for $T_{\text {eff }}$ below $2,000 \mathrm{~K}$ (and to $T_{\text {eff }} \approx 1,000 \mathrm{~K}$ ). This requires the opacities and chemical equilibria data down to $T \approx 500 \mathrm{~K}$. We have added the $\mathrm{CH}_{4}$ opacity to our VAEBM72 including 9 molecules as mentioned in Sect.3. Here, however, we assume that $\mathrm{CH}_{4}$ lines are well smeared out by the high line density due to the transitions from numerous vibrational states and/or by the pressure broadening, and thus $\mathrm{CH}_{4}$ is included as a pseudo-continuous opacity. In fact, the JOLA may be applicable to polyatomic molecules with complicated spectra while it is not to diatomic molecules with simple spectra, as we have noted in Sect.3. Another important opacity source at high density environment of very low mass stars is the CIA of $\mathrm{H}_{2}$, which dominates the whole infrared region by quasi-continuous absorption (Borysow in this volume). Also, convective energy transport is highly efficient up to the very surface of the atmosphere in cool dwarf stars. However, as the temperature gradient in the convective zone approaches to the adiabatic gradient at the high density of cool dwarf atmospheres, the convergence in radiative-convective equilibria is easily attained.

The predicted infrared spectra for model atmospheres of $T_{\text {eff }}=1,600 \mathrm{~K}$ are shown in Fig.7. The heavy solid line and the dashed line are the blanketed spectra from model atmospheres with and without $\mathrm{CH}_{4}$, respectively. Clearly, the $\mathrm{CH}_{4}$ opacity produces a serious effect upon the infrared spectrum. Especially, the strong $\nu_{3}$ band of methane at $3.3 \mu \mathrm{m}$ appears to be comparable with the $\nu_{1}$ and $\nu_{3}$ bands of water vapour at $2.7 \mu \mathrm{m}$. The $\mathrm{CH}_{4}$ opacity also modifies the atmospheric structure as to give an appreciable change of the $\mathrm{H}_{2} \mathrm{O}$ absorption. The thin solid line is the expected true continuum which can be observed if there are true windows in the spectrum and if they are resolved by very high resolution spectroscopy. It is interesting to observe that the expected peaking of the continuum flux at $1.6 \mu \mathrm{m}$ corresponding to the minimum of $\mathrm{H}^{-}$absorption does not appear, but broad depression centered at about $2.2 \mu \mathrm{m}$ appears instead. This depression is caused by the CIA of the $\mathrm{H}_{2}$ fundamental band, which is regarded as a continuous opacity rather than a line opacity in our models. Anyhow, this result clearly demonstrates the importance of the CIA at the high density and low temperature environment of brown dwarf atmospheres. Also, predicted spec- 


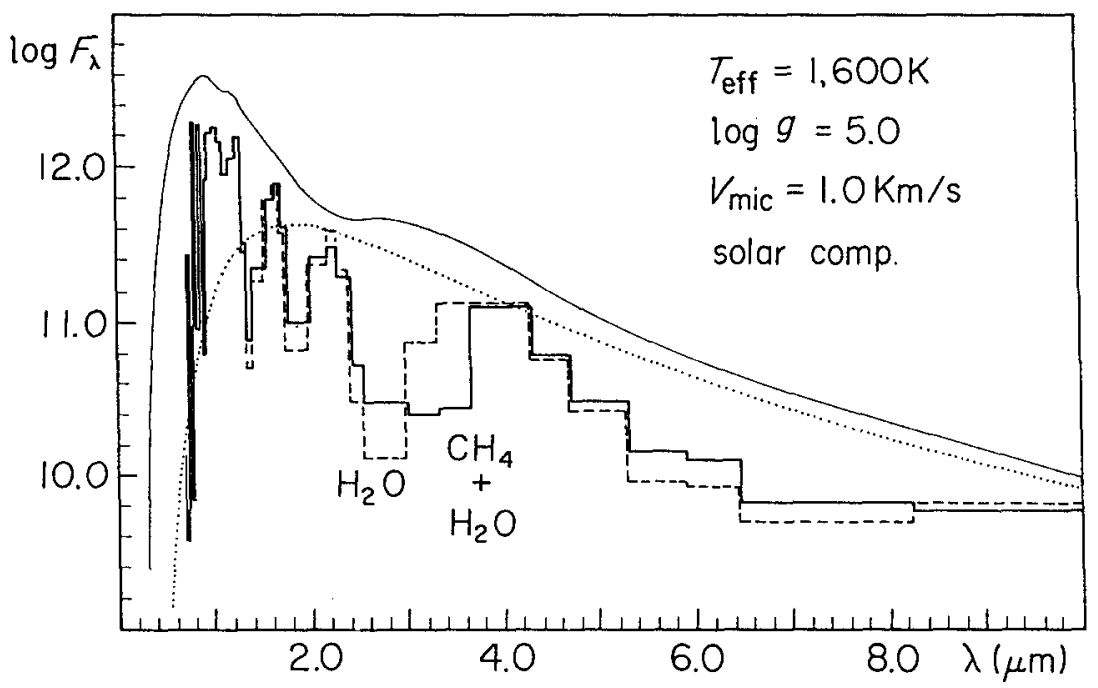

Fig. 7. Predicted infrared spectrum of brown dwarf $\left(T_{\text {eff }}=1,600 \mathrm{~K}\right)$ including $\mathrm{CH}_{4}$ (in addition to $\mathrm{H}_{2} \mathrm{O}, \mathrm{CO}, \mathrm{OH}, \mathrm{TiO}$ etc) as opacity source is shown by the heavy solid line, and predicted spectrum based on a model without $\mathrm{CH}_{4}$ by the dashed line for comparison. Clearly, a prominent spectroscopic signature of brown dwarf is the appearance of the $\mathrm{CH}_{4} \nu_{3}$-band at $3.3 \mu \mathrm{m}$. The continuous flux including $\mathrm{H}_{2}$ CIA is shown by the thin solid line, indicating that the CIA is a dominant opacity source as is evidenced by a dip at $2: 2 \mu \mathrm{m}$. The black body radiation of $T=1,600 \mathrm{~K}$ is shown by the dotted line.

tra based on the model atmosphere shows large deviation from the blackbody radiation of $T=1,600 \mathrm{~K}$ shown by the dotted line. Especially, there appear large excesses of flux at around $1 \mu \mathrm{m}$. There is still a possibility that these excesses are artifacts of neglecting some opacity sources in this region. At the same time, it is true that the infrared region beyond $1.2 \mu \mathrm{m}$ is highly opaque in the brown dwarf atmospheres not only due to the combined effect of the $\mathrm{H}_{2} \mathrm{O}$ and $\mathrm{CH}_{4}$ opacities (see Fig.3) but also due to the quasi-continuous $\mathrm{H}_{2}$ CIA.

The results outlined above may have some implications on the current searches for brown dwarfs. So far, many candidates for brown dwarfs have been proposed, but none was confirmed to be a true brown dwarf. Most of these candidates are bright brown dwarfs that are still warm on the cooling tracks and they are difficult to be distinguished from $M$ dwarfs that occupy the same region on the HR diagram. Future increase of sensitivity in observations will finally make it possible to observe brown dwarfs below the main-sequence limiting luminosity in the region of $L<6.10^{-5} L_{\odot}$ and $T_{\text {eff }}<1,800 \mathrm{~K}$ (Burrows et al. 1993). But here, accurate determination of the luminosity (or distance) is again difficult. On the other hand, our models revealed that $\mathrm{CH}_{4}$ can be observed only in the brown dwarfs with $T_{\text {eff }}$ below $1,800 \mathrm{~K}$, and the $\mathrm{CH}_{4} 3.3 \mu \mathrm{m}$ band can be the most reliable spectroscopic criterion in confirming brown dwarfs. Another interesting result is that brown dwarfs are not necessarily very red but rather may show 
large flux excesses around $1 \mu \mathrm{m}$. Also, brown dwarfs may not be bright at the $L$-band $\left(\lambda_{0} \approx 3.5 \mu \mathrm{m}\right)$ of IR photometry because of the strong $\mathrm{CH}_{4}$ absorption, and brown dwarf searches by this band may not be effective.

Our exploratory model atmosphere of brown dwarfs clearly shows the importance of molecular opacities for predicting reliable spectroscopic and photometric properties of such very cool and dim objects, and many problems remain to be worked out. First, a more accurate line-by-line database for $\mathrm{CH}_{4}$ will be required in the near future when the presence of such substellar objects will be confirmed. The latest version of the HITRAN database already includes 47,415 lines of $\mathrm{CH}_{4}$ (Rothman et al. 1992) but, since these are mostly low excitation atmospheric lines, it would require another effort to include many highly excited lines of this complicated molecule. Second, opacity sources around $1 \mu \mathrm{m}$ are crucial for understanding the photometric properties of brown dwarfs, and more careful investigations are needed. Third, in view of the importance of CIA at high density, improved opacites such as those by Borysow \& Frommhold (1990) are most welcome and will hopefully be extended to other molecules such as $\mathrm{N}_{2}$. Fourth, grain formation and its effect on opacities may be important (Lunine et al. 1989).

\section{Further Explorations on Stellar Atmospheres}

Now, if the case of brown dwarf is the last exploration in model stellar atmospheres, has the role of approximate molecular opacities ended? This may to some extent be true if we confine our attention to the classical model atmospheres. In fact, the classical model atmospheres may have been well sophisticated by now. Then, one important role of the accurate classical model may be to use it as a reference by which deviations of real atmosphere from the classical model atmosphere can be examined. For example, it appeared difficult to reproduce the best empirical model of the Sun by theoretical modelling within the framework of the classical atmosphere even by the use of the accurate line opacities (Kurucz 1979). Deviations of the order of $100 \mathrm{~K}$ appear in deeper layers as well as in the very surface layers, and this may be due to the limitation of the classical model atmosphere itself. Such a detailed test of model atmospheres by the empirical model, however, may be difficult for stars other than the Sun.

More recently, direct diagnoses of stellar atmospheres have been made possible by the high resolution spectroscopy of line profiles (e.g. Dravins 1987; Gray 1988). Further, molecular lines served to provid additional information on the solar atmosphere (Grevesse \& Sauval in this volume) and to extend the similar analysis to very cool stars (Tsuji 1991). All these observations revealed the presence of somewhat ordered velocity fields in stellar atmospheres rather than the chaotic turbulent motion as assumed in the classical atmospheres. An attempt to model such a non-thermal velocity structure has been done recently by Nordlund \& Dravins (1990), who have solved detailed 3D time-dependent radiation hydrodynamics of granular convection in solar type stars. For extending such a modelling of the dynamical atmospheres to very cool stars, molecular opacities 
should play a significant role, and approximate opacities such as by the band model method may be the best choice for such a purpose. In fact, it may be too complicating to introduce detailed line-by-line opacities based on a large database in such a computation which in itself is already highly complicated.

Also, larger deviations from the classical model atmosphere will occur in the surface layer and outer atmosphere where various kinds of non-thermal and nonequilibrium processes should take place. Because of the low temperatures, this is also the place where various molecules are formed, and molecules such as $\mathrm{CO}$ may play an important role in determining the atmospheric structure of cool stars because of their characteristic cooling function (Ayres 1981; Kneer 1983). The effect of $\mathrm{CO}$ on the dynamics of the solar atmosphere has actually been followed by time-dependent hydrodynamic codes first with a simple two-frequency opacity (Muchmore \& Ulmschneider 1985), and then with more detailed ODF opacity for CO (Muchmore et al. 1988). These analyses revealed that even the simple two-frequency opacity gave the correct physics for most cases, and the ODF method may be preferred if high accuracy is required. In the atmospheres of cool stars, other molecules will play a similar role and the case of $\mathrm{SiO}$ in the outer atmospheres of cool giant stars has been discussed already (Muchmore et al. 1987). For extending a similar analysis to other molecules and to other stellar environments, approximate treatments for molecular opacities will again be attractive because of their simplicity and flexibility. Also, accuracy of the approximate opacities may be sufficient for these purposes, since the major contribution to the cooling function is represented by Eq.(20) which depends little on the treatments of opacities as noted in Sect.3.

Further, new observations are still revealing unexpected new phenomena. An interesting case is the IRAS carbon stars that show silicate emission: the presence of the silicate emission implies the presence of an oxygen-rich environment $(\mathrm{C} / \mathrm{O}<1)$ while a carbon star characterized by the carbon-rich chemistry $(\mathrm{C} / \mathrm{O}>1)$ must be there at the same time. One interesting possibility is to assume a presence of an accretion disk composed of the oxygen-rich material around a classical carbon star (Lloyd Evans 1990; Lambert et al. 1990). Also, the cool accretion disk will be an important ingredient in understanding the physical structures of a wide variety of objects through young stellar systems such as $\mathrm{T}$ Tau stars to evolved systems such as cataclysmic variables. For modelling such cool accretion disks, molecular opacities should be important and approximate methods for molecular opacities will find wide applications in such new explorations.

As may be evident from the few examples noted above, explorations on stellar atmospheres continue and approximate approach for molecular opacities will remain to be an important tool in such endeavours.

\section{Concluding Remarks}

Briefly summarizing, the band model such as the VAEBM provides a simple method to estimate the ODF based on a few spectroscopic constants, instead of 
using a large database composed of millions of lines. As a result, molecular opacities can be expressed by simple analytical formulae which are very convenient in actual applications of various kind. Of course, accuracy of such approximate methods cannot be very high as compared with that of the methods directly based on a line-by-line database. However, there are many problems that can be attacked with the accuracy to be realized by the approximate methods. Especially, approximate molecular opacities played a major role in exploring model atmospheres of almost all the types of cool stars including brown dwarfs, and will play a similar role if we are to go beyond the classical atmospheres in our explorations of stellar atmospheres.

At the same time, approximate opacities can also be used for some practical purposes, but the limitations must be well recognized. For example, we found a temperature difference as large as $100 \mathrm{~K}$ between the model atmospheres based on the VAEBM and those based on the OS opacities. This difference is probably significant from the view point of constructing self-consistent model atmospheres. However, if we remember the fact that a similar difference is also found between the best empirical model and theoretical one for the case of the Sun, it may anyhow be difficult to know the true temperatures in stellar atmospheres with an accuracy better than $100 \mathrm{~K}$ by the present classical model atmospheres. Thus, there can be a viewpoint that the model atmospheres based on the approximate opacities can well be used for practical purposes such as abundance determinations by the quantitative analysis of high resolution stellar spectra. On the other hand, it is more difficult for approximate opacities to predict accurate emergent flux which depends directly on the details of the absorption coefficients. Especially, it is too crude to apply the predicted fluxes based on the JOLA for diatomic molecules to a direct interpretation of observed stellar fluxes. The over-simplification of the JOLA can largely be relaxed by the band model approach, but still very high accuracy cannot be expected in general. For polyatomic molecules, however, it is interesting to clarify the conditions by which the line opacity can be regarded as a pseudo-continuum due to sufficient line overlappings.

The present review necessarily focused attention to the approximate methods for molecular opacities, but this is not to suggest that the approximate molecular opacities can in any way replace the accurate opacities. Rather, we hoped to show that very simple and flexible methods may sometimes be useful, beside the accurate opacities. In fact, we should probably employ different approaches to investigate such complex systems as stellar atmospheres and stellar environments, and it may be useful to have various methods for treating molecular opacities differing in elaboration and in accuracy. Since the recent progresses in molecular science and in computer technology finally made it possible to realize the accurate opacities on a line-by-line basis, we are now in such a favourable situation as to be able to apply these high accuracy opacities to the problems that may truly require them. Under such a situation, it should still be most important to develope an accurate and complete line database for all the molecules of astrophysical interests. Such a database will be important not only as the opacity 
data but also as the basic data for interpreting a wide range of observations from accurate photometry to very high resolution spectroscopy. Also, only by such a database, the accuracy of approximate methods can be examined.

\section{References}

Alexander D.R., Augason G.C., Johnson H.R., 1989, Astrophy. J., 345, 1014

Auman J.R., 1967a, in Colloquium on Late-Type Stars, ed. M.Hack, p.313, Astron. Obs. Trieste

Auman J.R., 1967b, Astrophys. J. Suppl., 14, 171.

Ayres T.R., 1981, Astrophys. J., 244, 1064

Borysow A., Frommhold L., 1990, Astrophys. J. Letter, 348, L41

Brown J.A., Johnson H.R., Alexander D.R., Cutright L., Sharp C.M., 1989, Astrophys. J. Suppl., 71, 623

Burrows A., Hubbard W.B., Saumon D., Lunine J.I., 1993, Astrophys. J., 406, 158

Carbon D.F., 1979, Ann. Rev. Astron. Astrophys., 17, 513.

Dravins D., 1987, Astron. Astrophys., 172, 200 \& 211

Golden S.A., 1967, J. Quant. Spectrosc. Rad. Transf., 7, 225

Golden S.A., 1969, J. Quant. Spectrosc. Rad. Transf., 9, 1067

Goody R.M., 1964, Atmospheric Radiation I. Theoretical Basis, Oxford Univ. Press, Gray D.F., 1988, Lectures on Spectral-Line Analysis: F, G, and $K$ Stars, The Publisher Grenfell T.C., 1974, Astron. Astrophys., 31, 303

Gustafsson B., Bell R.A., Eriksson K., Nordlund A., 1975, Astron. Astrophys., 42, 407 Herzberg G., 1945, Molecular Spectra and Molecular Structure II. Infrared and Raman Spectra of Polyatomic Molecules, D.van Nostrand Co. Inc.

Kneer F., 1983, Astron. Astrophys., 128, 311

Kurucz R.L., 1979, Astrophys. J. Suppl., 40, 1

Johnson H.R., 1973, Astrophys. J., 180, 81

Johnson H.R., Bernat A.P., Krupp B.M., 1980, Astrophys. J. Suppl., 42, 501

Jørgensen U.G., Johnson H.R., Nordlund A., 1992, Astron. Astrophys., 261, 263

Jørgensen U.G., 1993, Rev. Mexicana Astron. Astrof., 23, 195

Lambert D.L., Hinkle K.H., Smith V.V., 1990, Astron. J., 99, 1612

Lloyd Evans T., 1990, Mon. Not. R. Astr. Soc., 243, 349

Ludwig C.B., 1967, Study on Exhaust Plume Radiation Predictions, GDC-DBE67-021, General Dynamics

Lunine J.I., Hubbard W.B., Burrows A., Wang Y-P., Garlow K., 1989, Astrophys. J., 338, 314

Muchmore D.O., Kurucz R.L., Ulmschneider P., 1988, Astron. Astrophys., 201, 138

Muchmore D.O., Nuth III J.A., Stencel R.E., 1987, Astrophys. J. Letters, 315, L141

Muchmore D.O., Ulmschneider P., 1985, Astron. Astrophys., 142, 393

Nordlund $\AA$., Dravins D., 1990, Astron. Astrophys., 228, 155

Plez B., Brett J.M., Nordlund $\AA$., 1992, Astron. Astrophys., 256, 551

Querci F., Querci M., Tsuji T., 1974, Astron. Astrophys., 31, 265

Rothman L.S. et al., 1992, J. Quant. Spectrosc. Rad. Transf., 48, 469

Saxner M., Gustafsson B., 1984, Astron. Astrophys., 140, 334

Schmid-Burgk J., Scholz M., Wehrse R., 1981, Mon. Not. R. Astr. Soc., 194, 383

Scholz M., Tsuji T., 1984, Astron. Astrophys., 130, 11

Tsuji T., 1966, Publ. Astron. Soc. Japan, 18, 127 
Tsuji T., 1967, in Colloquium on Late-Type Stars, ed. M.Hack, p.260, Astron. Obs. Trieste

Tsuji T., 1969, in Low Luminosity Stars, ed. S.S.Kumar, p.457, Gordon and Breach

Tsuji T., 1971, Publ. Astron. Soc. Japan, 23, 553

Tsuji T., 1976, Publ. Astron. Soc. Japan, 28, 543

Tsuji T., 1978, Astron. Astrophys., 62, 29

Tsuji T., 1984, Astron. Astrophys., 134, 24

Tsuji T., 1991, Astron. Astrophys., 245, 203

Watanabe T., Kodaira K., 1978, Publ. Astron. Soc. Japan, 30, 21

Wehrse R., 1975, Astron. Astrophys., 39, 169

Zeidler-K.T. E.-M., Koester D., 1982, Astron. Astrophys., 113, 173 